\title{
Long non-coding RNA HOTTIP promotes renal cell carcinoma progression through the regulation of the miR-615/IGF-2 pathway
}

\author{
QIFEI WANG ${ }^{*}$, GUANGZHEN WU*, ZHIWEI ZHANG, QIZHEN TANG, WEI ZHENG, \\ XIAOCHI CHEN, FENG CHEN, QUANLIN LI and XIANGYU CHE
}

\author{
Department of Urology, The First Affiliated Hospital of Dalian Medical University, Dalian, Liaoning 116011, P.R. China
}

Received November 29, 2017; Accepted July 27, 2018

DOI: $10.3892 /$ ijo.2018.4539

\begin{abstract}
Emerging evidence has indicated that long non-coding RNA (lncRNA) HOXA transcript at the distal tip (HOTTIP) regulates cell growth, differentiation, apoptosis and cancer progression. However, the expression and function of HOTTIP in the progression of renal cell carcinoma (RCC) remain largely unknown. In this study, we investigated the role of the lncRNA HOTTIP in RCC. The expression levels of HOTTIP in RCC tissues and cell lines were determined by RT-qPCR. The association between HOTTIP expression and clinicopathological characteristics and prognosis was analyzed in patients with RCC from the TCGA database. Loss-of- function assays were designed and conducted to verify the oncogenic function of HOTTIP in RCC progression. Luciferase assay was performed to explore the mechanisms of the miRNA-lncRNA sponge. The results revealed that HOTTIP expression was upregulated in RCC. An increased HOTTIP expression in RCC was associated with a larger tumor size and a higher clinical stage, lymph node metastasis and vascular invasion. Additionally, patients RCC with a high HOTTIP expression had a significantly shorter overall survival (OS) and disease-free survival (DFS). HOTTIP knockdown significantly inhibited cell proliferation, migration and invasion, and increased the apoptosis of RCC cells in vitro. Mechanistic analyses revealed that HOTTIP functioned as a competing endogenous RNA (ceRNA) for hsa-miR-615-3p, and led to the derepression of its endogenous target, insulin-like growth factor-2 (IGF-2), which is a protein hormone that exerts a stimulatory effect on tumor cell growth. miR-615 inhibition reversed the suppressive effects of HOTTIP knockdown on RCC cell progression. HOTTIP regulated IGF-2 expression
\end{abstract}

Correspondence to: Dr Quanlin Li or Dr Xiangyu Che, Department of Urology, The First Affiliated Hospital of Dalian Medical University, Dalian, Liaoning 116011, P.R. China

E-mail: quanlin_li123@163.com

E-mail: chexiang_yu@163.com

*Contributed equally

Key words: renal cell carcinoma, long non-coding RNA, HOXA transcript at the distal tip, insulin-like growth factor 2 in a miR-615-dependent manner in RCC cells. In addition, IGF-2 expression was significantly upregulated in the RCC specimens and a positive association between the expression of HOTTIP and IGF-2 in RCC tissues was detected. The effect of HOTTIP was abolished by the siRNA-mediated silencing of IGF-2 in RCC cells. On the whole, this study demonstrates, for the first time, at least to the best of our knowledge, that the HOTTIP/miR-615/IGF-2 axis plays an important role in RCC progression and potentially contributes to the improvement of RCC diagnosis and therapy.

\section{Introduction}

Renal cell carcinoma (RCC) is one of the most common malignancy affecting adults, and the incidence of RCC has increased over the past two decades (1). The mortality rate of patients with RCC appears to be increasing each year (2). In addition, patients with RCC respond poorly to conventional chemotherapy and radiotherapy treatment (3). Thus, further understanding of the molecular mechanisms responsible for the development and progression of RCC is of particular importance.

It is well known that protein-coding genes account for $<2 \%$ of the total genome DNA, whereas a large number of the human genome can be transcribed into non-coding RNAs (ncRNAs) (4-6). Recently, new members of the family of ncRNAs (>200 nucleotides) with limited or no protein-coding potential, long ncRNAs (IncRNAs) have been extensively studied in both physiological and pathological processes $(7,8)$. Accumulating evidence has indicated that lncRNAs are emerging as key molecules in human malignancies, disease progression and metastasis $(5,9,10)$. However, the role of lncRNAs in RCC remains unclear.

The HOXA transcript at the distal tip (HOTTIP) lncRNA, located at the 5' end of the HOXA cluster, has recently been functionally characterized (11). HOTTIP primarily targets WDR5/MLL complexes across HOXA by directly binding the adaptor protein, WD repeat-containing protein 5 (WDR5), leading to histone $\mathrm{H} 3$ lysine 4 trimethylation and the gene transcription of several 5' HOXA genes (12). It has been demonstrated that HOTTIP plays a major role in tumor progression (13). An increased HOTTIP expression has been reported in various types of cancer, such as non-small cell lung cancer (14) gastric cancer (15), colorectal cancer (16) and prostate cancer (17). In these tumors, HOTTIP may serve as a potential oncogene 
and may be a poor prognostic factor for patients. However, the functions of HOTTIP and its potential mechanisms of action in RCC have not yet been fully elucidated.

Recently, a class of lncRNAs, referred to as competing endogenous RNAs (ceRNAs), has been characterized, including lncARSR and Linc00152 (18). For instance, lnc-MD1, the first identified ceRNA involved in myogenesis, has been shown to control muscle cell differentiation by competing for the binding of miR-133 and miR-135 (18). The function of ceRNAs has been described as a novel regulatory mechanism of RNAs (19), and this is a newly proposed mechanism that describes a crosstalk among lncRNAs, mRNAs and their shared microRNAs (miRNAs or miRs). ceRNAs protect mRNAs by acting as molecular sponges for miRNAs that specifically repress the target mRNAs. This hypothesis suggests that lncRNAs, mRNAs and pseudogenes can communicate with each other by competing for common miRNA response elements.

In this study, we demonstrate that HOTTIP expression was significantly upregulated in RCC and was associated with a larger tumor size and a higher clinical stage, lymph node metastasis vascular invasion, and a shorter overall survival (OS) and disease-free survival (DFS). The inhibition of HOTTIP suppressed cell proliferation, migration and invasion in RCC. In addition, IFG-2 was found to be a direct target gene of HOTTIP. HOTTIP directly bound to miR-615 and effectively acted as a sponge for miR-615 to modulate the suppression of IFG-2. To the best of our knowledge, this study provides the first evidence that the HOTTIP/miR-615/IFG-2 axis plays an important role in RCC tumorigenesis and progression and may thus have diagnostic and therapeutic potential for use as a target in RCC.

\section{Materials and methods}

Ethics statement. The use of tissues for this study was approved by the Medical Ethics Committee of Dalian Medical University. Due to the retrospective nature of the study, the Ethics Committee waived the need of written informed consent by the patients. All the samples were anonymous.

Clinical samples. Tumor specimens were collected from patients with primary RCC, who underwent surgery at the Department of Urology, the First Affiliated Hospital of Dalian Medical University (Dalian, China) from August, 2009 to January, 2016. The cohort consisted of 57 patients, from whom fresh tumor samples coupled with adjacent non-tumorous renal tissues $5-10 \mathrm{~cm}$ away from the tumor edge were obtained and subjected to HOTTIP and insulin-like growth factor-2 (IGF-2) mRNA and protein expression analysis. All the fresh specimens were stored at $-80^{\circ} \mathrm{C}$ until use.

TCGA data analysis. The lncRNA expression profiles and corresponding clinical information of the patients with RCC were obtained from The Cancer Genome Atlas (TCGA) data portal (up to May 27, 2016; https://cancergenome.nih.gov/). After excluding the data without complete survival information, a total of 529 patients with RCC and 72 paired non-tumor renal samples were enrolled in this study. We also downloaded the detailed clinical information of the patients with RCC, including age, sex, tumor grade, AJCC cancer stage, etc.
Cell culture and transfection. The human RCC cell lines (A-498, 786-O, Caki-1, Caki-2 and ACHN), the normal renal epithelial cells HK-2, 293T cell line were obtained from the American Tissue Culture Collection (ATCC, Manassas, VA, USA). The cells were cultured in RPMI-1640 or DMEM (Gibco/Thermo Fisher Scientific, Waltham, MA, USA) supplemented with 10\% FBS (Gibco-BRL, Gaithersburg, MD, USA), 100 U/ml penicillin and $100 \mathrm{mg} / \mathrm{ml}$ streptomycin (Gibco/Thermo Fisher Scientific) in humidified air of $5 \% \mathrm{CO}_{2}$ at $37^{\circ} \mathrm{C}$.

Small interfering RNAs (siRNAs) specifically targeting human HOTTIP, IGF-2 and negative control siRNA (siNC) were synthesized by Life Technologies/Thermo Fisher Scientific. The target HOTTIP siRNA sequence was 5'-UGGGAACCCG CUAUUUCACUCUAUU-3'. The target IGF-2 siRNA sequence was 5'-GGGUUUUCUUUUGACUUAUTT-3'. miR-615-3p mimics (5'-UCCGAGCCUGGGUCUCCCUCUU-3'), miR-615-3p inhibitor (5'-ACCGAGUCAGGGAUACCCA CAA-3'), miR-615-3p mimic negative control (5'-UUCUCGA ACGUGUCACGUUUU-3') and miR-615-3p inhibitor negative control (5'-CAGUACUUUUGUGUAGUACAA-3') were chemically synthesized by RiboBio (Guangzhou, China). The cells were grown in 6-well plates to $70 \%$ confluence and transfected with the siRNAs $(50 \mathrm{nM})$, mimics $(20 \mathrm{nM})$ or inhibitors $(20 \mathrm{nM})$ for $48 \mathrm{~h}$ using Lipofectamine 2000 (Invitrogen/Thermo Fisher Scientific) according to the manufacturer's instructions. The transfection efficiency was examined by reverse transcription-quantitative PCR (RT-qPCR).

Cell proliferation assay. Cell proliferation was measured by MTT assay. Briefly, the cells were seeded into 96-well plates at 1,000 cells/well and incubated for 1, 2, 3 and 4 days. Subsequently, $20 \mu \mathrm{l}$ of MTT (5 mg/ml; Sigma, St. Louis, MO, USA) were added to each well and followed by incubation at $37^{\circ} \mathrm{C}$ for $4 \mathrm{~h}$. Viable cells were evaluated by absorbance measurements at $490 \mathrm{~nm}$ using a microplate reader (Molecular Devices, Sunnyvale, CA, USA).

Flow cytometric analysis. To analyze cell cycle progression, the cells fixed and incubated with RNase A $(0.25 \mathrm{mg} / \mathrm{ml}$; Sigma) followed by treatment with propidium iodide (PI; Nanjing KeyGen Biotech Co., Ltd., Nanjing, China). The cell cycle was analyzed using a FACSCalibur flow cytometer (Beckman Coulter, Brea, CA, USA). For apoptosis assay, the cells were seeded at a density of $1 \times 10^{6}$ cells/well and cultured in 6-well plates at $37^{\circ} \mathrm{C}$ and transfected with HOTTIP siRNA. After $48 \mathrm{~h}$, the cells, including, the adhesive and floating cells were harvested and washed twice with cold PBS and re-suspended in $1 \mathrm{X}$ binding buffer (Invitrogen/Thermo Fisher Scientific). Subsequently, $10 \mu 1$ of Annexin V-FITC (Invitrogen/Thermo Fisher Scientific) and $5 \mu \mathrm{l}$ of PI were added to each cell suspension. The fluorescence of the stained cells was then analyzed by flow cytometry.

Matrigel invasion assay. A Matrigel invasion chamber (BD Biosciences, Bedford, MA, USA) was used for the Matrigel invasion assay. In brief, the cells were seeded into the top chamber with serum-free medium. The lower chamber was filled with $10 \%$ FBS. The cells were incubated for $48 \mathrm{~h}$ and the cells that did not invade through the pores were removed using a cotton swab. The cells on the lower surface of the membrane were stained with crystal violet at room temperature for $20 \mathrm{~min}$ 
Table I. Sequences of primers used for RT-qPCR.

\begin{tabular}{lll}
\hline Name & \multicolumn{1}{c}{ Forward primer (5'-3') } & \multicolumn{1}{c}{ Reverse primer (5'-3') } \\
\hline HOTTIP & CCTAAAGCCACGCTTCTTTG & TGCAGGCTGGAGATCCTACT-3 \\
IGF-2 & AGACCCTTTGCGGTGGAGA & GGAAACATCTCGCTCGGACT \\
pri-miR-615 & GCAAGTCGAGCATTTTACCTGC & GCCATGTGTCCACTGAAATGTG \\
pre-miR-615 & ACACTCCAGCTGGGTCCGAGCCTGGGTCTC & TGGTGTCGTGGAGTCG \\
GAPDH & CAGCCTCAAGATCATCAGCA & TGTGGTCATGAGTCCTTCCA \\
U6 & ATTGGAACGATACAGAGAAGATT & GGAACGCTTCACGAATTTG
\end{tabular}

HOTTIP, HOXA transcript at the distal tip ; IGF-2, insulin-like growth factor 2; GAPDH, glyceraldehyde 3-phosphate dehydrogenase.

(Sigma) and counted under a dissecting microscope (Olympus Optical, Tokyo, Japan). The experiment was repeated 3 times.

$R T$ - $q P C R$. RNA was extracted from cells or clinical samples using TRIzol reagent (Invitrogen/Thermo Fisher Scientific). Subsequently, $1 \mu \mathrm{g}$ RNA was reverse transcribed into cDNA using PrimeScript RT-polymerase (Takara, Dalian, China). Quantitative PCR (qPCR) was performed using the SYBR Premix Ex Taq $^{\mathrm{TM}}$ kit (Takara). qPCR for mRNA expression was conducted as follows: Initial denaturation at $95^{\circ} \mathrm{C}$ for $30 \mathrm{sec}$, followed by 40 cycles of annealing at $95^{\circ} \mathrm{C}$ for $15 \mathrm{sec}$ and extension at $60^{\circ} \mathrm{C}$ for $30 \mathrm{sec}$. qPCR for miRNA expression was conducted as follows: Initial denaturation at $95^{\circ} \mathrm{C}$ for $3 \mathrm{~min}$, followed by 40 cycles of annealing at $95^{\circ} \mathrm{C}$ for $12 \mathrm{sec}$ and extension at $62^{\circ} \mathrm{C}$ for $60 \mathrm{sec}$. GAPDH was used as an internal control. The results were quantified using the $2^{-\Delta \Delta \mathrm{Cq}}$ method (20). The primers were synthesized by Ribobio Co. The corresponding primers are listed in Table I.

Western blot analysis. Western blot analyses were performed as previously described (21). In brief, the cells were lysed by a radio immunoprecipitation assay (RIPA) buffer and extracted using Mammalian Protein Extraction Reagent (both from Thermo Fisher Scientific). The concentration of the protein was determined using the Bradford Protein Assay kit (Beyotime Institute of Biotechnology). Protein lysates $(30-50 \mu \mathrm{g})$ were separated on a $10 \%$ SDS/PAGE gel and transferred onto polyvinylidene fluoride membranes (PVDF; Millipore, Billerica, MA, USA). The membranes were then blocked with $5 \%$ non-fat milk at room temperature for $1.5 \mathrm{~h}$, and the membranes were then incubated with primary antibody at $4^{\circ} \mathrm{C}$ overnight, followed by $1 \mathrm{~h}$ of incubation with anti-mouse $\mathrm{IgG} /$ horseradish peroxidase-conjugated secondary antibody (sc-2380; Santa Cruz Biotechnology, Dallas, TX, USA) at room temperature. Immunocomplexes were visualized by chemiluminescence using ECL (Santa Cruz Biotechnology). The relative band intensity was calculated using ImageJ software (version 1.50; National Institutes of Health, Bethesda, MD, USA). The following antibodies and dilutions were used: IGF-2 (1:2,000; cat. no. sc-293176, mouse mAb), GAPDH (1:2,000; cat. no. sc-47724, mouse mAb) (both from Santa Cruz Biotechnology).

Luciferase reporter assays. To determine whether IGF-2 is a direct target of miR-615, Luciferase reporter vectors (control), or vectors containing wild-type (pGL3-IGF-2-3'-UTR-Full or mutated (pGL3-IGF-2-3'-UTR-Mut) 3'-UTRs of IGF-2 mRNA (Shanghai GenePharma Co., Ltd.) were co-transfected into 293T cells withmiR-615mimics, usingLipofectamine2000. Toconfirm whether HOTTIP can interact with miR-615 HOTTIP-WT/ HOTTIP-MUT were examined through PCR and a mutagenesis kit. The fragments, including the predicted binding sites, were cloned into a pmirGLO vector (Promega, Madison, WI, USA) as pmirGLO-HOTTIP-WT/pmirGLO-HOTTIP-MUT plasmids. All the plasmids were constructed by GeneChem (Shanghai, China). pmirGLO-HOTTIP-WT/pmirGLO-HOTTIP-MUT were co-transfected with miR-615 mimics into $293 \mathrm{~T}$ cells. At $48 \mathrm{~h}$ post-transfection, the cells were harvested and then assayed for reporter gene activities using the Dual-Luciferase Reporter Assay System (Promega) according to the manufacturer's instructions.

miRNA target gene prediction. To identify the possible molecular mechanisms of action of the miRNA and the miRNA target, we retrieved putative miRNA-mRNA interactions by prediction algorithms from TargetScan (http://www. targetscan.org/vert_71/).

Statistical analysis. All statistical analyses were performed using the SPSS 17.0 software package (SPSS, Chicago, IL, USA). The significance of differences between groups was estimated by one-way ANOVA, followed by LSD post-hoc tests. The results are reported as the means $\pm \mathrm{SD}$. A Chi-square test were used to compare the associations between HOTTIP expression and the patient clinicopathological parameters. Wilcoxon signed-rank test was used to compare the differences of HOTTIP expression between normal and cancer from TCGA database. Survival analyses were performed using the Kaplan-Meier method and survival differences were estimated using the log-rank test. The correlations of different genes were assessed by Pearson's correlation analysis. Statistical significance was assigned at $\mathrm{P}<0.05$. All experiments were performed at least 3 times with triplicate samples.

\section{Results}

Association between HOTTIP expression and patient clinicopathological characteristics. Initially, RT-qPCR was used to examine HOTTIP expression in the 57 matched tissues and a panel of RCC cell lines (A-498, 786-O, Caki-1, Caki-2 and 
A

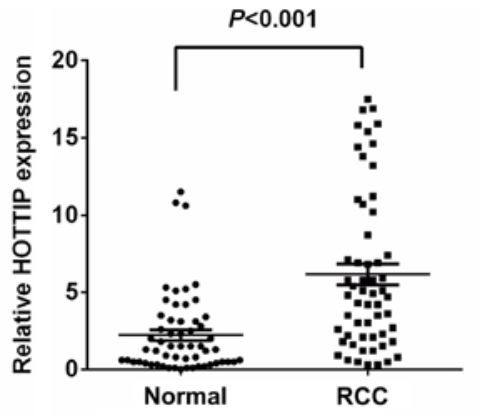

C

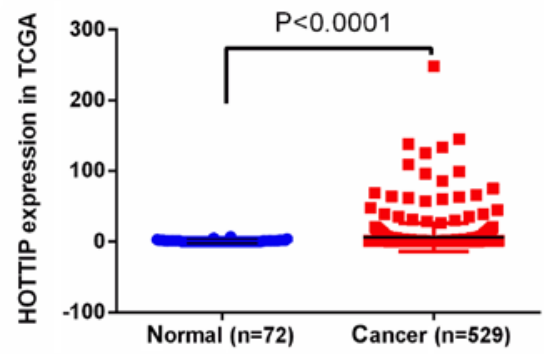

B

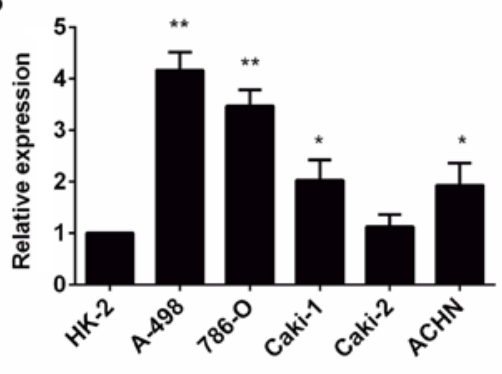

D

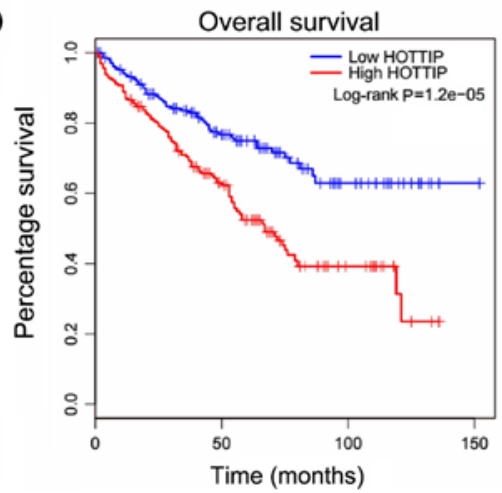

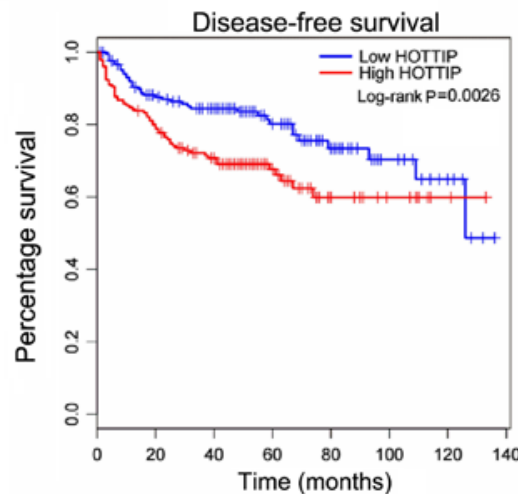

Figure 1. HOTTIP expression and its association with the clinical parameters in renal cancer tissues. (A) Expression of HOTTIP in RCC from our clinical specimens. (B) Results of RT-qPCR showing the expression levels of HOTTIP in RCC cell lines. (C) HOTTIP RNAseq expression data were retrieved from the TCGA database. The Wilcoxon signed-rank test was used for the comparison. (D) The overall survival (OS) and disease-free survival (DFS) curve for patients with RCC with low vs. high HOTTIP expression (Kaplan-Meier method). ${ }^{*} \mathrm{P}<0.05,{ }^{* * *} \mathrm{P}<0.01$. RCC, renal cell carcinoma.

ACHN). The results revealed that HOTTIP expression was significantly increased in all renal cancer tissues compared with the matched normal kidney tissues (Fig. 1A). HOTTIP expression was also significantly increased in four RCC cell lines (A-498, 786-O, Caki-1 and ACHN), but not in the Caki-2 cell lines, compared with that in the normal prostate epithelial cell line, HK-2 (Fig. 1B, P<0.05). The A-498 and 786-O cells exhibited the highest HOTTIP expression. Thus, the A-498 and 786-O cells were used as a model to perform the following experiments to examine the effects of HOTTIP on the proliferation, apoptosis, migration and invasion of RCC cells in vitro.

We then investigated the association between HOTTIP expression with the clinicopathological characteristics of patients with RCC from the TCGA database. Consistently, the quantification of the HOTTIP levels demonstrated significantly higher expression levels of HOTTIP in the tumors compared with the matched adjacent normal tissues (Fig. 1C, $\mathrm{P}<0.05)$. According to the median of the HOTTIP expression value in TCGA, the samples were divided into the high and low expression groups. As presented in Table II, an increased HOTTIP expression was significantly associated with a high
TNM stage $(\mathrm{P}=0.003)$, tumor size $(\mathrm{P}=0.001)$, and pathological grade $(\mathrm{P}=0.005)$. However, HOTTIP protein expression was not associated with other clinicopathological characteristics such as sex, lymph node involvement and distant metastasis. In addition, patients with RCC with high HOTTIP expression levels had a shorter OS and DFS than those with low HOTTIP expression levels (Fig. 1D), as shown by Kaplan-Meier survival analysis. These results demonstrated that a high expression level of HOTTIP was associated with a poor prognosis in RCC. The upregulation of HOTTIP may thus play an important role in RCC development and progression.

Effect of HOTTIP on the viability, cell cycle progression, apoptosis and invasion of renal cancer cells. Subsequently, the knockdown of HOTTIP using siRNA in the A-498 and 786-O cells was performed to evaluated the biological role of HOTTIP in RCC cell proliferation, migration and invasion. The results of RT-qPCR performed at $48 \mathrm{~h}$ post-transfection confirmed the silencing efficiency. Following transfection with HOTTIP siRNA (si-HOTTIP), HOTTIP expression was downregulated in the A-498 and 786-O cells (Fig. 2A). The results of MTT 
A

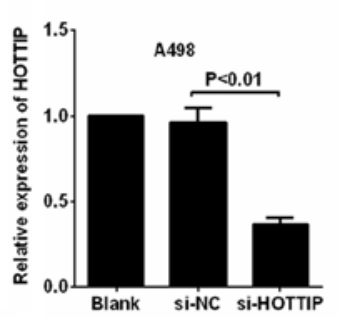

B

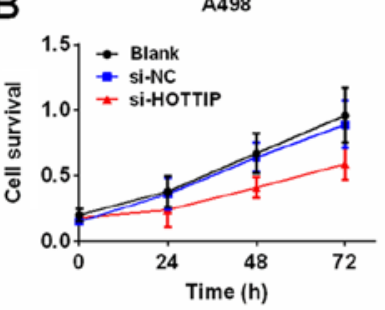

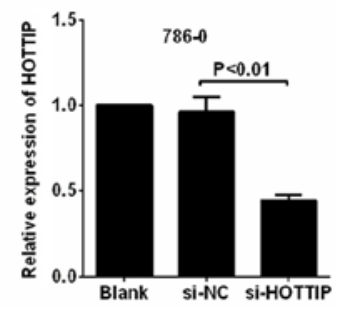

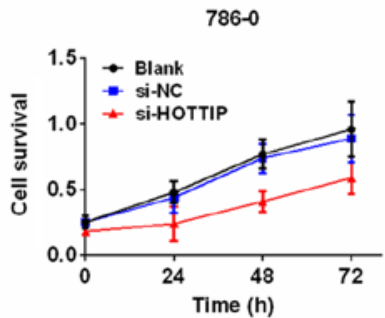

D
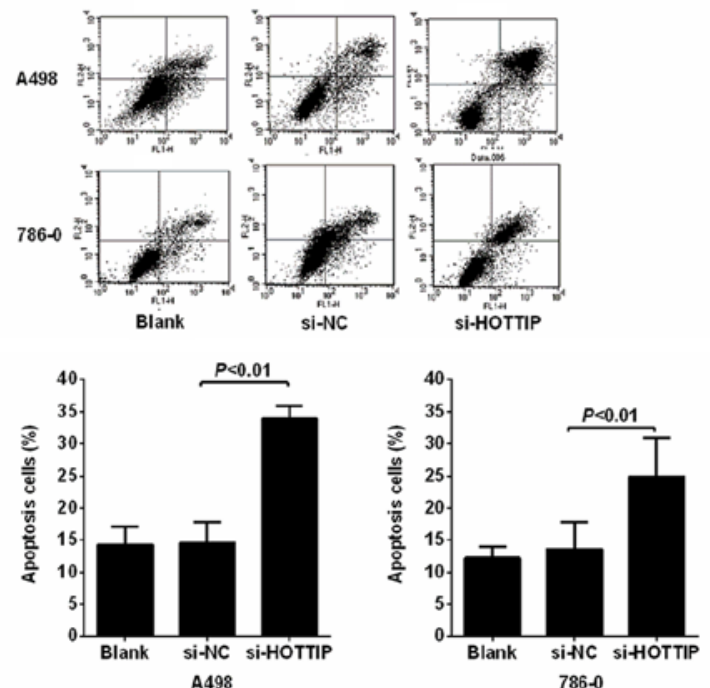

C
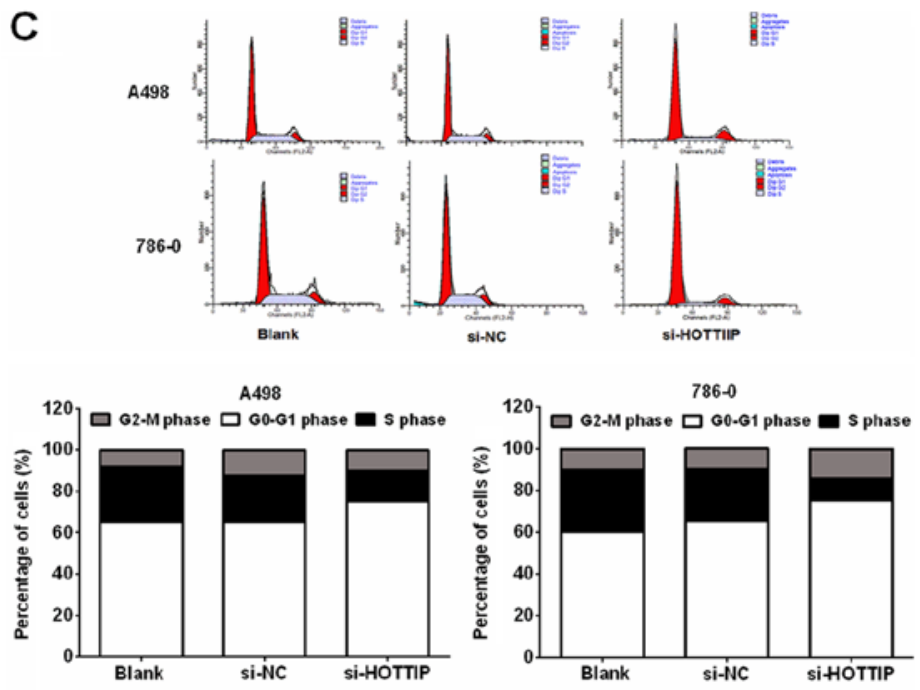

E
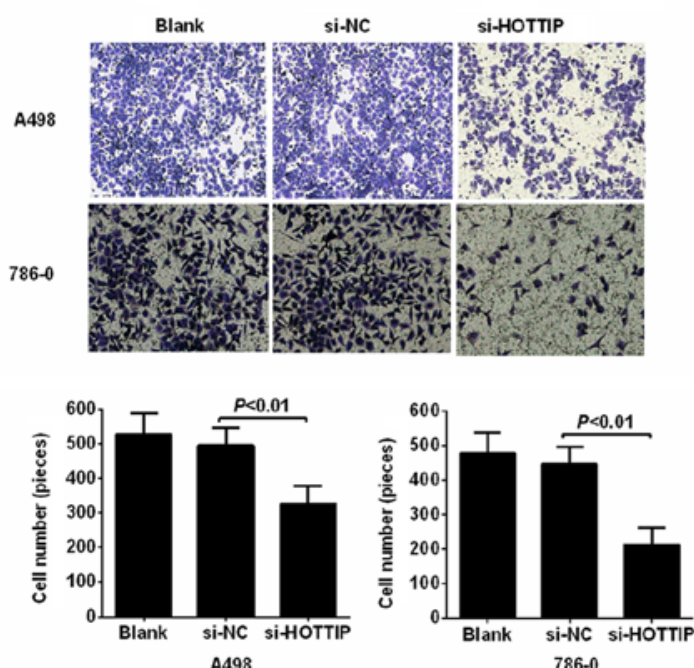

Figure 2. Effect of HOTTIP knockdown on renal cancer cell function. A-498 and 786-O cells were transiently transfected with either si-HOTTIP (a final concentration of $50 \mathrm{nM}$ ) or control (si-NC). (A) Results of RT-qPCR showing the mRNA expression of HOTTIP in A-498 and 786-O cells which significantly decreased following transfection with HOTTIP siRNA compared with the control cells. (B) Results of MTT assay showing that HOTTIP knockdown inhibited the proliferation of A-498 and 786-O cells. (C) Cell cycle analysis of the cells in which HOTTIP was knocked down. The A-498 and 786-O cells were transfected with si-HOTTIP or control shRNA for $48 \mathrm{~h}$, then fixed and stained with propidium iodide, and analyzed by flow cytometry. (D) Apoptosis of cells were measured using a FACScan flow cytometer after staining with FITC-Annexin V and propidium iodide. (E) Matrigel-based invasion assay was performed using chambers with $10 \%$ FBS as a chemoattractant. Representative images and quantitative analysis are presented. Data are presented as the means \pm SD of 3 independent experiments performed in triplicate. si-NC, negative control siRNA; si-HOTTIP, siRNA against HOTTIP.

assay then revealed that cell viability was suppressed following transfection with si-HOTTIP compared with the negative control in both the A-498 and 786-O cell lines (Fig. 2B). To further investigate the observed inhibition of proliferation following HOTTIP knockdown, we compared the cell cycle profiles of the cells in which HOTTIP was knocked down by flow cytometry. The results revealed that the inhibition of HOTTIP led to a decrease in the number of cells in the $\mathrm{S}$ phase and an increase in the percentage of cells in the G0/G1 phase (Fig. 2C).

Some lncRNAs have been reported to play important roles in tumor cell apoptosis $(22,23)$. Thus, in this study, to examine the effects of HOTTIP on RCC cell apoptosis, the percentage of apoptotic cells was measured by flow cytometry with PI and Annexin V double staining. The results revealed that the apoptotic population was notably increased in the A-498 and 786-O cells in which HOTTIP was knocked down compared with the controls (Fig. 2D). Subsequently, we examined the effects of HOTTIP knockdown on cell invasion. We performed a Matrigel assay, as shown in Fig. 2E. The knockdown of HOTTIP induced a significant decrease in invasiveness compared with the controls. Collectively, these results clearly indicated that the downregulation of HOTTIP markedly suppressed the proliferation and invasion of RCC cells in vitro, and promoted cell apoptosis.

Reciprocal repression of HOTTIP and miR-615. As stated in the Introduction, increasing numbers of studies have suggested that lncRNAs may act as ceRNAs in regulating the biological functions of miRNAs. In this study, to further explore the underlying mechanisms of action of HOTTIP and its role in the development of RCC, we first examined a set of miRNAs that were predicted to bind HOTTIP using TargetScan. Bioinformatics analysis revealed that the HOTTIP transcript contained putative binding sites for miR-615-3p (Fig. 3A). To 
Table II. Association of HOTTIP expression with clinicopathological characteristics of patients with renal cell carcinoma from the TCGA database.

\begin{tabular}{|c|c|c|c|c|}
\hline \multirow[b]{2}{*}{ Covariate } & \multirow[b]{2}{*}{ Classification } & \multicolumn{3}{|c|}{ HOTTIP } \\
\hline & & Low expression, n (\%) & High expression, $\mathrm{n}(\%)$ & P-value \\
\hline Age, years & $\begin{array}{l}<61 \\
\geq 61\end{array}$ & $\begin{array}{r}102(19.28) \\
93(17.58)\end{array}$ & $\begin{array}{l}179(33.84) \\
155(29.30)\end{array}$ & 0.775 \\
\hline Sex & $\begin{array}{l}\text { Female } \\
\text { Male }\end{array}$ & $\begin{array}{r}71(13.42) \\
124(23.44)\end{array}$ & $\begin{array}{l}114(21.55) \\
220(41.59)\end{array}$ & 0.596 \\
\hline Pathological grade & $\begin{array}{c}\text { I-II } \\
\text { III-IV }\end{array}$ & $\begin{array}{r}104(19.77) \\
89(16.92)\end{array}$ & $\begin{array}{l}137(26.05) \\
196(37.26)\end{array}$ & 0.005 \\
\hline Lymph node involvement & $\begin{array}{l}\text { NO-N1 } \\
\text { N2-NX }\end{array}$ & $\begin{array}{r}88(16.64) \\
107(20.23)\end{array}$ & $\begin{array}{l}151(28.54) \\
183(34.59)\end{array}$ & 0.986 \\
\hline Distant metastasis & $\begin{array}{c}\text { M0 } \\
\text { M1-MX }\end{array}$ & $\begin{array}{c}162(30.74) \\
33(6.26)\end{array}$ & $\begin{array}{r}258(48.96) \\
74(14.04)\end{array}$ & 0.139 \\
\hline Tumor T (size) & $\begin{array}{l}\text { T1-T2 } \\
\text { T3-T4 }\end{array}$ & $\begin{array}{c}145(27.41) \\
50(9.45)\end{array}$ & $\begin{array}{l}200(37.81) \\
134(25.33)\end{array}$ & 0.001 \\
\hline TNM stage & $\begin{array}{c}\text { I-II } \\
\text { III-IV }\end{array}$ & $\begin{array}{r}135(25.67) \\
59(11.22)\end{array}$ & $\begin{array}{l}187(35.55) \\
145(27.57)\end{array}$ & 0.003 \\
\hline
\end{tabular}

Values in bold indicate statistical significance $(\mathrm{P}<0.05)$ as determined by the Chi-square test.

A
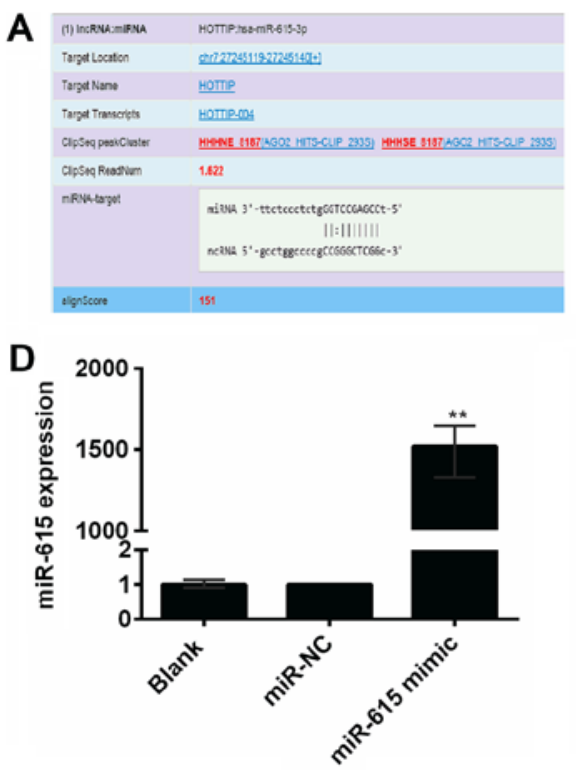

$\mathbf{F}$

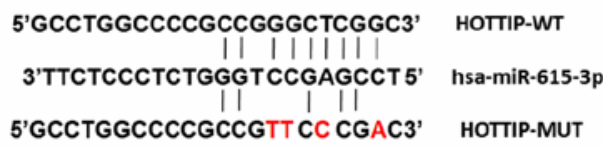

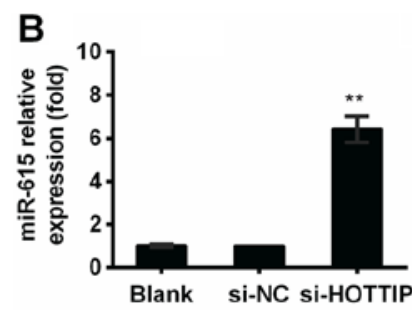

Blank si-NC si-HOTTIP
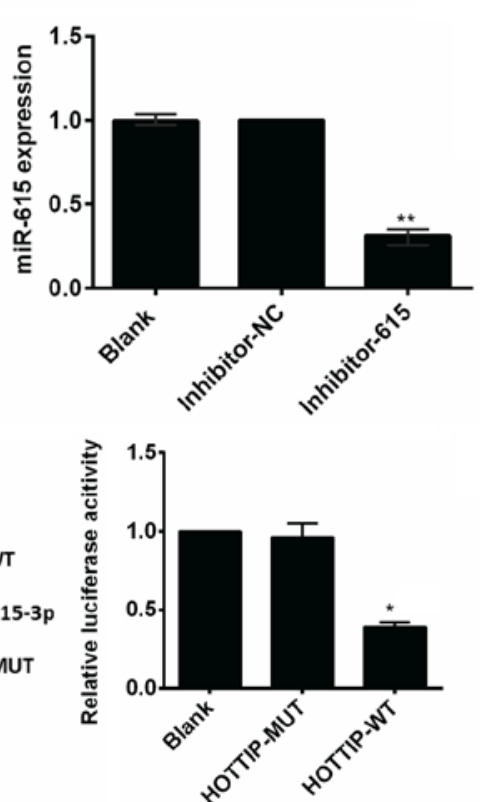

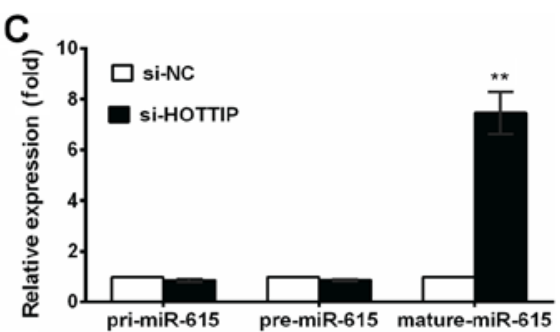

E

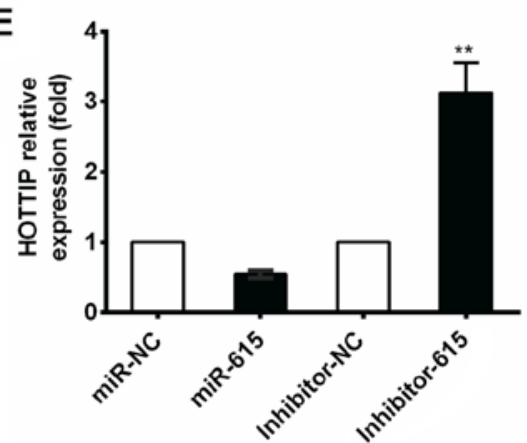

Figure 3. Reciprocal repression of HOTTIP and miR-615. (A) starBase prediction indicated that miR-615 could bind to HOTTIP. (B and C) A-498 cells were transiently transfected with either si-HOTTIP or the control (si-NC). (B) Results of RT-qPCR showing the expression of miR-615 in A-498 cells. (C) RT-qPCR analysis was performed to determine the effects of HOTTIP on the expression levels of pri-miR-615, pre-miR-615 and mature miR-615. (D) RT-qPCR analysis was performed to determine the transfection efficiency of miR-615 mimic or inhibitor (a final concentration of $20 \mathrm{nM}$ ). (E) RT-qPCR analysis of HOTTIP expression in A-498 cells transfected for $48 \mathrm{~h}$ with miR-NC, miR-615 mimic, miR-NC inhibitor and miR-615 inhibitor. (F) The luciferase reporter assay demonstrated that the overexpression of miR-615 reduced the intensity of fluorescence in $293 \mathrm{~T}$ cells transfected with the HOTTIP-WT vector, while it had no effect on the HOTTIP-MUT vector. ${ }^{*} \mathrm{P}<0.05,{ }^{* *} \mathrm{P}<0.01$. si-NC, negative control siRNA; si-HOTTIP, siRNA against HOTTIP. 

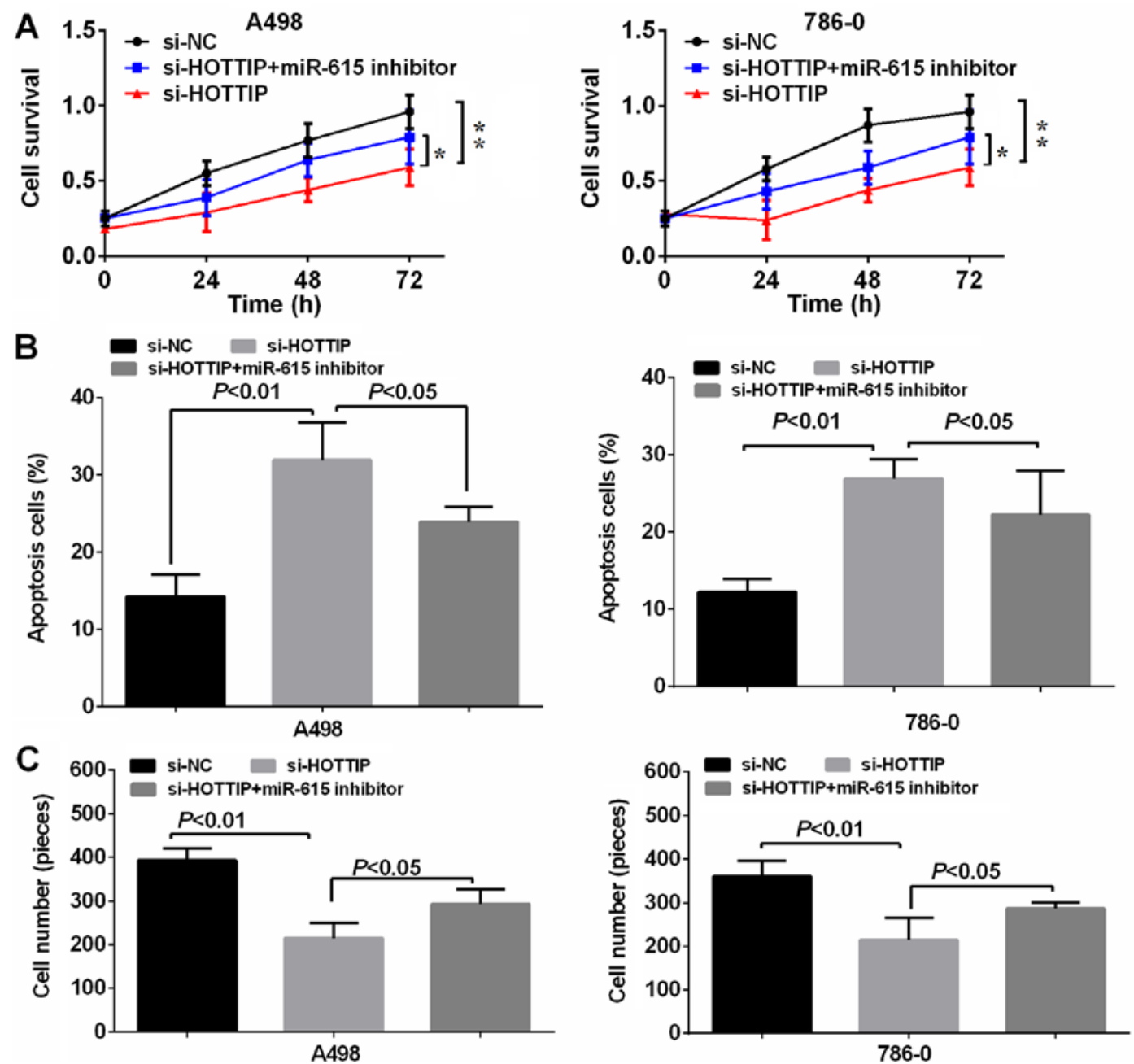

Figure 4. HOTTIP activity is partially exerted through its negative regulation of miR-615. si-HOTTIP and miR-615 inhibitor were co-transfected into A-498 and $786-\mathrm{O}$ cells. (A) Cell viability was detected by MTT assay at 24,48 and $72 \mathrm{~h}$ after transfection. ${ }^{*} \mathrm{P}<0.05,{ }^{* * *} \mathrm{P}<0.01$. (B) Cell apoptosis was measured using a FACScan flow cytometer and the quantification of apoptosis is shown. (C) Cell migration was analyzed by Matrigel assay $48 \mathrm{~h}$ after transfection. The invasiveness of the cells was evaluated by counting the cells that had migrated through the Transwell membranes with Matrigel. si-NC, negative control siRNA; si-HOTTIP, siRNA against HOTTIP.

further examine this hypothesis, we performed RT-qPCR assay to detect the changes in miR-615 expression in the A-498 and 786-O cells transfected with si-HOTTIP, and found that the expression of miR-615 was increased following transfection with si-HOTTIP (Fig. 3B). To explore the underlying mechanisms of the regulation of miR-615 expression by HOTTIP, we examined the effects of HOTTIP on the expression levels of pri-miR-615 and pre-miR-615 by RT-qPCR. The expression levels of pri-miR-615 or pre-miR-615 were not affected by si-HOTTIP (Fig. 3C), suggesting that the HOTTIP-mediated regulation of miR-615 was likely achieved through a post-transcriptional mechanism. To determine whether miR-615 negatively and reciprocally regulated HOTTIP, miR-615 mimic or inhibitor were transfected into the A-498 cells (Fig. 3D), and a significant inhibition of HOTTIP expression by miR-615 mimic was observed. Conversely, the miR-615 inhibitor markedly increased HOTTIP expression (Fig. 3E).

Moreover, we also performed a dual-luciferase reporter assayto investigate the potential interaction of HOTTIP with miR-615. We obtained HOTTIP-WT/HOTTIP-MUT through PCR and a mutagenesis kit. The fragments, including the predicted binding sites, were cloned into a pmirGLO vector as pmirGLOHOTTIP-WT/pmirGLO-HOTTIP-MUT. The recombinant plasmids were transiently co-transfected with a miR-615 mimic and scrambled oligonucleotides into 293T cells. Our results demonstrated miR-615 overexpression reduced the luciferase activity of the pmirGLO-HOTTIP-WT reporter vector, but not that of the empty vector or pmirGLO-HOTTIP-MUT reporter vector (Fig. 3F). These results suggested that the interaction between HOTTIP and miR-615 had reciprocal effects.

The oncogenic functions of HOTTIP are partially mediated through the negative regulation of miR-615. In order to verify the reciprocal repression of HOTTIP and miR-615, and to determine whether HOTTIP exerts its biological functions through miR-615, we performed a rescue experiment. We co-transfected si-HOTTIP and miR-615 inhibitor into the A-498 and 786-O cell lines. The results revealed that the miR-615 inhibitor significantly reversed the suppressive effects exerted by the knockdown of HOTTIP on cell proliferation and invasion, and the promoting effects on the apoptosis of A-498 and 786-O cells (Fig. 4), suggesting that HOTTIP plays its oncogenic role in RCC cells through miR-615. 
A

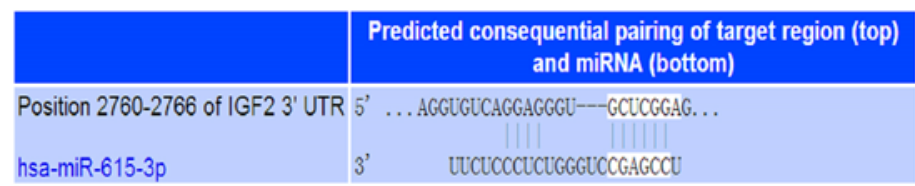

B

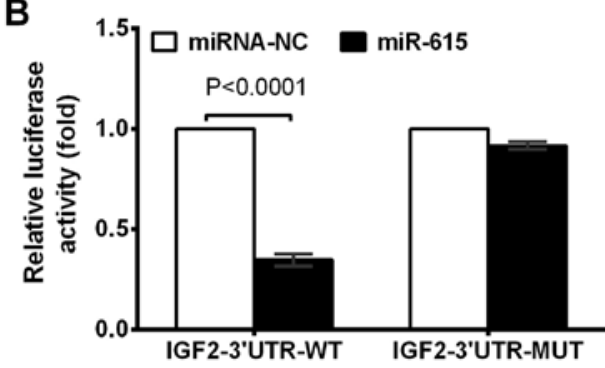

D

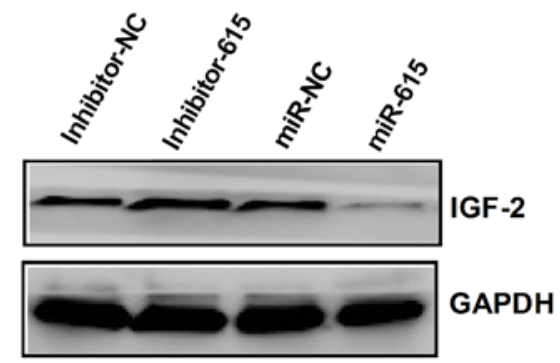

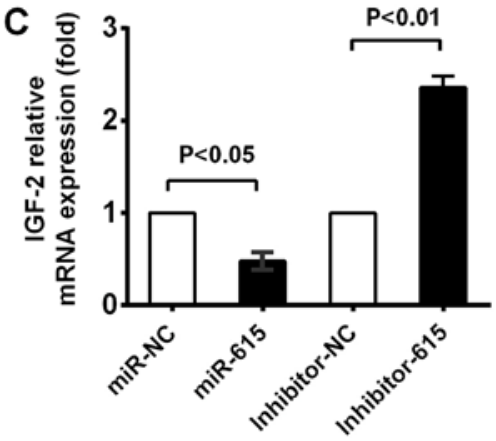

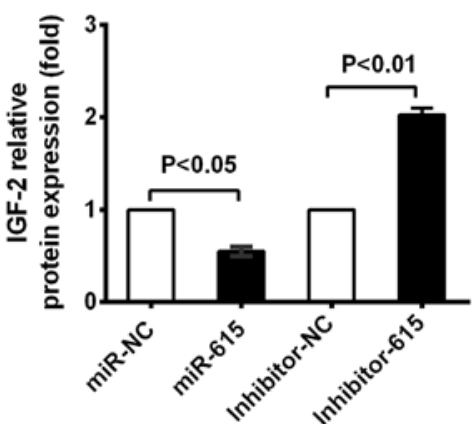

Figure 5. IGF-2 is a direct target of miR-615. (A) Bioinformatics analysis predicted the miR-615 binding sites in IGF-2. (B) Luciferase reporter assay of 293 cells transfected with the IGF-2-3'-UTR-WT or mutant reporter vector containing mutation (IGF-2-3'-UTR-MUT) in the miR-615 binding sites and miR-615 mimic. (C and D) A-498 cells were transfected with miR-615 mimic $(20 \mathrm{nM})$ or inhibitor $(20 \mathrm{nM})$ for $48 \mathrm{~h}$. (C) Results of RT-qPCR showing the mRNA expression of IGF-2 and (D) results of western blot analysis showing the protein expression of IGF-2 in cells.

IGF-2 is a direct target of miR-615. Based on the abovementioned results, we aimed to identify the main target genes of miR-615, which were predicted using the TargetScan website. IGF-2 was predicted to be one of the targets of miR-615 (Fig. 5A). As it has been reported that IGF-2 is involved in cancer progression $(24,25)]$, in this study, we thus selected IGF-2 as our target gene to study the mechanisms responsible for the HOTTIP-induced progression of RCC mediated by miR-615. The results of luciferase reporter assay revealed that miR-615 mimics significantly reduced luciferase activity compared to miR-615 NC in the wild-type IGF-2 construct, whereas the luciferase activity in the IGF-2-MUT construct exhibited no change in the cells transfected with miR-615 mimics and miR-615 NC group (Fig. 5B), suggesting that miR-615 directly binds to IGF-2-3'-UTR. Consistent with the results of reporter assay, the IGF-2 mRNA and protein expression levels were decreased in the presence of miR-615 mimic in the A-498 cells. Conversely, when miR-615 expression was inhibited, the opposite effects were observed (Fig. 5C and D). These results indicated that IGF-2 was a direct target of miR-615.

HOTTIP regulates IGF-2 expression in an miR-615-dependent manner in RCC cells. According to the ceRNA concept, lncRNAs can act as ceRNAs to carry out their regulatory functions (26). In this study, we found that HOTTIP shared regulatory miR-615 binding sites with IGF-2 (Figs. 3A and 5A).
To determine whether HOTTIP interacts with miR-615 to regulate IGF-2 expression, we examined the mRNA and protein levels of IGF-2. The inhibition of HOTTIP significantly decreased the expression of IGF-2 at the mRNA and protein level, and this effect was significantly attenuated by miR-615 inhibitor (Fig. 6A and B), indicating that HOTTIP regulated IGF-2 expression in a miR-615-dependent manner.

Furthermore, we assessed the correlation between HOTTIP mRNA and IGF-2 expression in RCC tissues. Compared with normal kidney tissues, the mRNA levels of IGF-2 were significantly higher in renal cancer tissues (Fig. 6C). In addition, we found that HOTTIP expression significantly and positively correlated with IGF-2 expression (two-sided Pearson' s correlation, $r=0.314, \mathrm{P}<0.0001$; Fig. 6D).

To further examine whether HOTTIP carries out its biological functions through IGF-2, we performed rescue experiments by inhibiting IGF-2 expression in the cells in which HOTTIP was knocked down (Fig. 6E). MTT assay revealed that cell growth was reduced in the RCC cells in which HOTTIP was knocked down, whereas si-IGF-2 partially reversed the reduction of the cell proliferative ability (Fig. 6E).

\section{Discussion}

lncRNAs have been found to be involved in many biological processes, including carcinogenesis $(27,28)$. In RCC, several 
A

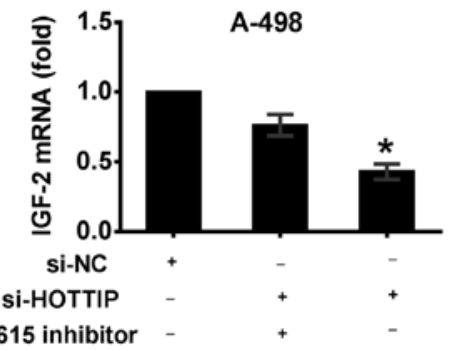

B

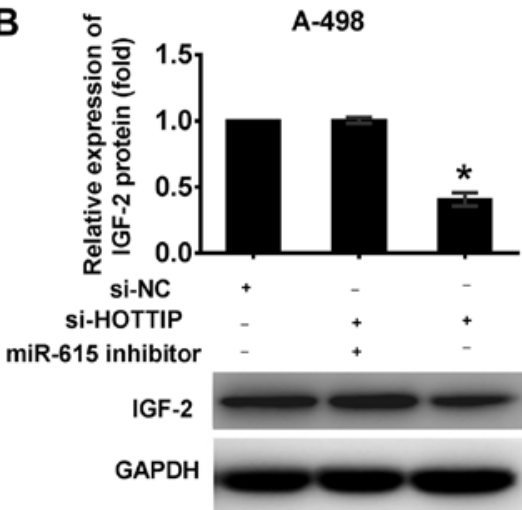

C

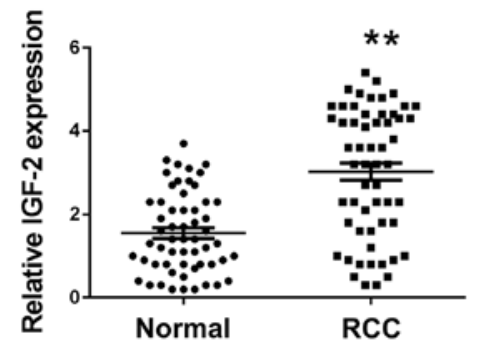

E

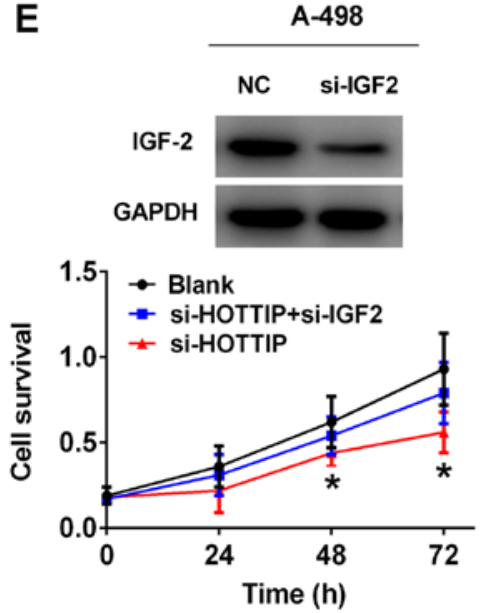

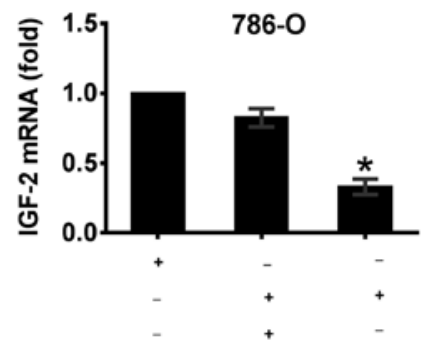

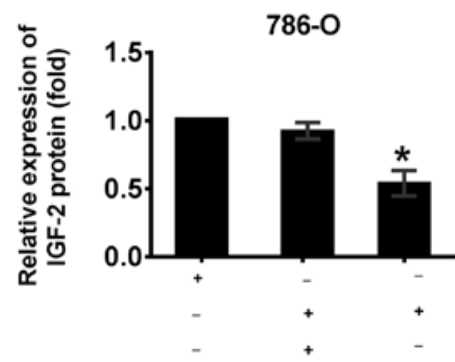

IGF-2
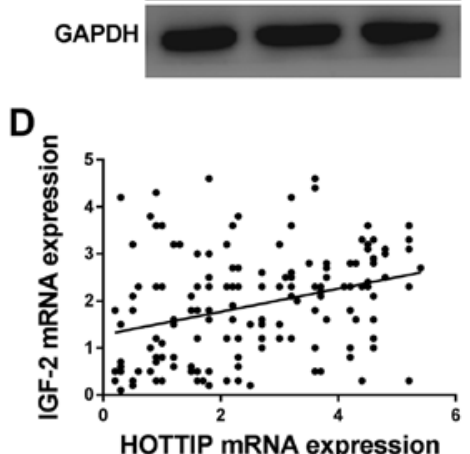

HOTTIP mRNA expression

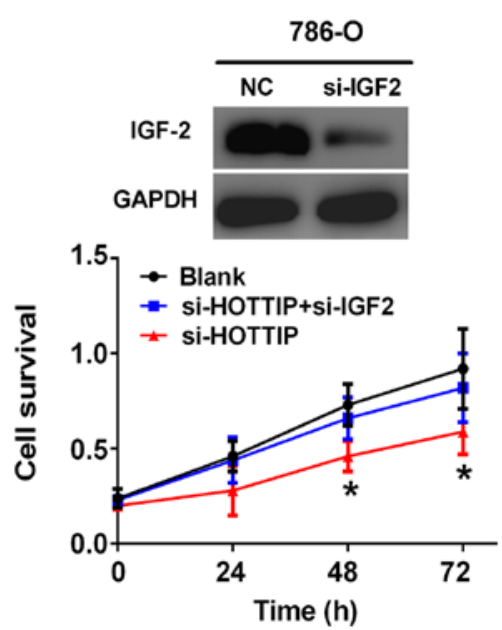

Figure 6. HOTTIP regulates IGF-2 expression in an miR-615-dependent manner in RCC cells. (A and B) si-HOTTIP (50 nM) and miR-615 inhibitor (20 nM) were co-transfected into A-498 and 786-O cell lines. (A) The expression level of IGF-2 was evaluated by RT-qPCR. (B) Western blot and densitometric analyses showing the protein expression of IGF-2 in A-498 and 786-O cells. (C) IGF-2 mRNA expression in normal kidney and matched renal cancer tissues. ${ }^{* *} \mathrm{P}<0.01$. (D) Pearson's correlation between HOTTIP mRNA and IGF-2 mRNA expression. (E) si-HOTTIP and si-IGF2 were co-transfected into A-498 and 786-O cells. The results of western blot analysis revealed that IGF-2 was effectively silenced by transfection with si-IGF in A-498 and 786-O cells. Cell viability was detected by MTT assay at 24,48 and $72 \mathrm{~h}$ after transfection. ${ }^{*} \mathrm{P}<0.05$. RCC, renal cell carcinoma.

lncRNAs have been reported to play important roles in tumorigenesis (29-32). For instance, Linc00152 is a positive prognostic factor in RCC. Hirata et al reported that MALAT1 promoted aggressive RCC through Ezh2 and interacted with miR-205 (32). In this study, we investigated the functional role and clinical significance of IncRNA HOTTIP in RCC. In several types of cancer, HOTTIP has been reported to be a potent oncogene $(14,17,33-35)$. In the present study, our results confirmed that HOTTIP expression was significantly higher in RCC tissues and cell lines. A high HOTTIP expression was also associated with a poor clinical outcome in patients with RCC. The downregulation of HOTTIP inhibited cell 
proliferation and invasion, and promoted the apoptosis of the RCC cells. In addition, we found that HOTTIP had a dual effect on cell proliferation and invasive ability. However, we did not determine whether the reduced invasive ability of the si-HOTTIP-transfected cells was due to reduced viability and/ or increased apoptosis. On the whole, however, our results suggest that HOTTIP functions as an oncogene and may thus be a potential therapeutic target for RCC.

Although HOTTIP has been suggested to act as an oncogene in different cancers, little is known about the underlying molecular mechanism. ceRNA theory is the most important theory regarding lncRNA, and lncRNA has been shown to be a sponge for regulating the expression and function of miRNA.

It has reported been that IncRNAs play a crucial role in multiple processes in cells by acting as ceRNAs to regulate miRNAs (9). In this study, we discovered that HOTTIP and miR-615 negatively regulated each other and were involved in the ceRNA regulatory network. HOTTIP knockdown increased miR-615 expression due to HOTTIP functioning as an endogenous miRNA sponge to bind to miR-615; the inhibition of HOTTIP abrogated this sponge, leading to an increase in miR-615 expression. Recent studies have indicated that miR-615 plays a tumor suppressive role in hepatoma cells (36), breast cancer cells (37) and pancreatic ductal adenocarcinoma (38). However, its role on RCC has not yet been investigated, at least to the best of our knowledge. In this study, we provide evidence that miR-615 targets not only protein-coding genes, but also the lncRNA HOTTIP. Hence, the identification of HOTTIP as an miR-615 target expands the repertoire of miR-615 targets. Furthermore, HOTTIP is able to repress miR-615, forming a reciprocal negative regulatory loop. These results provide further supporting evidence of the ceRNA regulatory network. Moreover, we found that HOTTIP carried out is oncogenic function through miR-615 in RCC cells. Taken together, these data strongly suggest that HOTTIP directly targets miR-615 and affects the biological characteristics of RCC cells by negatively regulating miR-615.

The IGF pathway is frequently activated in a variety of cancers (39). IGF-2 is maternally imprinted in the majority of normal tissues, with only the paternal allele expressed (40). In many tumors, however, this imprinting is lost, leading to the biallelic expression of the gene. The overproduction of the growth factor promotes the malignant behavior of tumor cells $(41,42)$. The ceRNA hypothesis indicates that lncRNAs may functions as ceRNAs by acting as endogenous decoys for miRNAs, which in turn affects the binding of miRNAs on their targets. In this study, the results of reporter assays indicated that IGF-2 was a direct target of miR-61 and that miR-615 downregulated the activity of the IGF-2-3'-UTR in RCC cells. Furthermore, the overexpression of miR-615 decreased IGF-2 expression in A-498 cells. In agreement with HOTTIP being a decoy for miR-615, we proved that the inhibition of HOTTIP significantly decreased IGF-2 expression, which was partially reversed in the presence of miR-615 inhibitor. Furthermore, we found that si-IGF-2 partially reversed the reduction of cell proliferation ability induced by si-HOTTIP. Therefore, the HOTTIP/miR-615/IGF-2 axis may play an important role in RCC progression.

In conclusion, in this study we demonstrated that HOTTIP contributed to carcinogenesis and functioned as a miRNA sponge to attenuate the endogenous function of miR-615, which negatively modulated IGF-2 expression in RCC. Due to this crucial role which HOTTIP plays in the progression of RCC, it may thus serve as a therapeutic target, as well as prognostic biomarker for RCC.

\section{Acknowledgements}

Not applicable.

\section{Funding}

No funding was received.

\section{Availability of data and materials}

All data generated or analyzed during this study are included in this published article.

\section{Authors' contributions}

QL and XC conceived and designed the research; QW, GW, ZZ and QT organized, analyzed and interpreted the data; QW, GW, ZZ, QT, WZ, XC and FC performed the experiments. QW and GW drafted and revised the manuscript; QL and XC supervised the study. All authors have read and approved the final manuscript.

\section{Ethics approval and consent to participate}

The use of tissues for this study was approved by the Medical Ethics Committee of Dalian Medical University. Due to the retrospective nature of the study, the Ethics Committee waived the need of written informed consent by the patients. All the samples were anonymous.

\section{Patient consent for publication}

Not applicable.

\section{Competing interests}

The authors declare that they have no competing interests.

\section{References}

1. Torre LA, Bray F, Siegel RL, Ferlay J, Lortet-Tieulent J and Jemal A: Global cancer statistics, 2012. CA Cancer J Clin 65: 87-108, 2015

2. Linehan WM: Genetic basis of kidney cancer: Role of genomics for the development of disease-based therapeutics. Genome Res 22: 2089-2100, 2012.

3. Porta C, Giglione $\mathrm{P}$ and Paglino $\mathrm{C}$ : Targeted therapy for renal cell carcinoma: Focus on 2 nd and 3rd line. Expert Opin Pharmacother 17: 643-655, 2016.

4. Guttman M, Amit I, Garber M, French C, Lin MF, Feldser D, Huarte M, Zuk O, Carey BW, Cassady JP, et al: Chromatin signature reveals over a thousand highly conserved large noncoding RNAs in mammals. Nature 458: 223-227, 2009.

5. Ponting CP, Oliver PL and Reik W: Evolution and functions of long noncoding RNAs. Cell 136: 629-641, 2009.

6. Wilusz JE, Sunwoo H and Spector DL: Long noncoding RNAs: Functional surprises from the RNA world. Genes Dev 23: 1494-1504, 2009. 
7. Fatica A and Bozzoni I: Long non-coding RNAs: New players in cell differentiation and development. Nat Rev Genet 15: 7-21, 2014.

8. St Laurent G, Wahlestedt C and Kapranov P: The Landscape of long noncoding RNA classification. Trends Genet 31: 239-251, 2015.

9. Mercer TR, Dinger ME and Mattick JS: Long non-coding RNAs: Insights into functions. Nat Rev Genet 10: 155-159, 2009.

10. Ntziachristos P, Abdel-Wahab O and Aifantis I: Emerging concepts of epigenetic dysregulation in hematological malignancies. Nat Immunol 17: 1016-1024, 2016.

11. Ong CT and Corces VG: Enhancer function: New insights into the regulation of tissue-specific gene expression. Nat Rev Genet 12: 283-293, 2011.

12. Xie H, Zhu D, Xu C, Zhu H, Chen P, Li H, Liu X, Xia Y and Tang W: Long none coding RNA HOTTIP/HOXA13 act as synergistic role by decreasing cell migration and proliferation in Hirschsprung disease. Biochem Biophys Res Commun 463 569-574, 2015

13. Lian Y, Cai Z, Gong H, Xue S, Wu D and Wang K: HOTTIP: A critical oncogenic long non-coding RNA in human cancers. Mol Biosyst 12: 3247-3253, 2016.

14. Sang Y, Zhou F, Wang D, Bi X, Liu X, Hao Z, Li Q and Zhang W: Up-regulation of long non-coding HOTTIP functions as an oncogene by regulating HOXA13 in non-small cell lung cancer. Am J Transl Res 8: 2022-2032, 2016.

15. Wang SS, Wuputra K, Liu CJ, Lin YC, Chen YT, Chai CY, Lin CS, Kuo KK, Tsai MH, Wang SW, et al: Oncogenic function of the homeobox A13-long noncoding RNA HOTTIP-insulin growth factor-binding protein 3 axis in human gastric cancer. Oncotarget 7: 36049-36064, 2016.

16. Ren YK, Xiao Y, Wan XB, Zhao YZ, Li J, Li Y, Han GS, Chen XB, Zou QY, Wang GC, et al: Association of long non-coding RNA HOTTIP with progression and prognosis in colorectal cancer. Int J Clin Exp Pathol 8: 11458-11463, 2015.

17. Zhang SR, Yang JK, Xie JK and Zhao LC: Long noncoding RNA HOTTIP contributes to the progression of prostate cancer by regulating HOXA13. Cell Mol Biol (Noisy-le-grand) 62: 84-88, 2016.

18. Cesana M, Cacchiarelli D, Legnini I, Santini T, Sthandier O, Chinappi M, Tramontano A and Bozzoni I: A long noncoding RNA controls muscle differentiation by functioning as a competing endogenous RNA. Cell 147: 358-369, 2011.

19. Ergun S and Oztuzcu S: Oncocers: ceRNA-mediated cross-talk by sponging miRNAs in oncogenic pathways. Tumour Biol 36: 3129-3136, 2015

20. Livak KJ and Schmittgen TD: Analysis of relative gene expression data using real-time quantitative PCR and the 2(- $\Delta \Delta \mathrm{C}(\mathrm{T}))$ method. Methods 25: 402-408, 2001.

21. Yang C, Cai J, Wang Q, Tang H, Cao J, Wu L and Wang Z: Epigenetic silencing of miR-130b in ovarian cancer promotes the development of multidrug resistance by targeting colonystimulating factor 1. Gynecol Oncol 124: 325-334, 2012.

22. Wang Y, Zhang L, Zheng X, Zhong W, Tian X, Yin B, Tian K and Zhang W: Long non-coding RNA LINC00161 sensitises osteosarcoma cells to cisplatin-induced apoptosis by regulating the miR-645-IFIT2 axis. Cancer Lett 382: 137-146, 2016.

23. He A, Liu Y, Chen Z, Li J, Chen M, Liu L, Liao X, Lv Z, Zhan Y, Zhuang C, et al: Over-expression of long noncoding RNA BANCR inhibits malignant phenotypes of human bladder cancer. J Exp Clin Cancer Res 35: 125, 2016.

24. Ma Y, Chen Y, Chen L, Liu Z, Ieong ML, Gao F and Huang W: Promotion of Insulin-like growth factor II in cell proliferation and epithelial-mesenchymal transition in hepatocellular carcinoma. J Cancer Res Ther 14: 844-850, 2018

25. Kessler SM, Haybaeck J and Kiemer AK: Insulin-like growth factor 2 - The oncogene and its accomplices. Curr Pharm Des 22 $5948-5961,2016$
26. Sumazin P, Yang X, Chiu HS, Chung WJ, Iyer A, Llobet-Navas D, Rajbhandari P, Bansal M, Guarnieri P, Silva J, et al: An extensive microRNA-mediated network of RNA-RNA interactions regulates established oncogenic pathways in glioblastoma. Cell 147: 370-381, 2011

27. Xie W, Yuan S, Sun Z and Li Y: Long noncoding and circular RNAs in lung cancer: Advances and perspectives. Epigenomics 8: 1275-1287, 2016

28. Shukla S, Zhang X, Niknafs YS, Xiao L, Mehra R, Cieślik M, Ross A, Schaeffer E, Malik B, Guo S, et al: Identification and Validation of PCAT14 as Prognostic Biomarker in Prostate Cancer. Neoplasia 18: 489-499, 2016.

29. Su H, Sun T, Wang H, Shi G, Zhang H, Sun F and Ye D: Decreased TCL6 expression is associated with poor prognosis in patients with clear cell renal cell carcinoma. Oncotarget 8: 5789-5799, 2017.

30. Wu Y, Tan C, Weng WW, Deng Y, Zhang QY, Yang XQ, Gan HL, Wang T, Zhang PP, Xu MD, et al: Long non-coding RNA Linc00152 is a positive prognostic factor for and demonstrates malignant biological behavior in clear cell renal cell carcinoma. Am J Cancer Res 6: 285-299, 2016.

31. Ellinger J, Alam J, Rothenburg J, Deng M, Schmidt D, Syring I, Miersch H, Perner S and Müller SC: The long non-coding RNA lnc-ZNF180-2 is a prognostic biomarker in patients with clear cell renal cell carcinoma. Am J Cancer Res 5: 2799-2807, 2015.

32. Hirata H, Hinoda Y, Shahryari V, Deng G, Nakajima K, Tabatabai ZL, Ishii $\mathrm{N}$ and Dahiya R: Long noncoding RNA MALAT1 promotes aggressive renal cell carcinoma through Ezh2 and interacts with miR-205. Cancer Res 75: 1322-1331, 2015.

33. Ye H, Liu K and Qian K: Overexpression of long noncoding RNA HOTTIP promotes tumor invasion and predicts poor prognosis in gastric cancer. Onco Targets Ther 9: 2081-2088, 2016.

34. Li F, Cao L, Hang D, Wang F and Wang Q: Long non-coding RNA HOTTIP is up-regulated and associated with poor prognosis in patients with osteosarcoma. Int J Clin Exp Pathol 8: 11414-11420, 2015

35. Chang S, Liu J, Guo S, He S, Qiu G, Lu J, Wang J, Fan L, Zhao W and Che X: HOTTIP and HOXA13 are oncogenes associated with gastric cancer progression. Oncol Rep 35: 3577-3585, 2016.

36. Chen Z, Wang X, Liu R, Chen L, Yi J, Qi B, Shuang Z, Liu M, Li X, Li S, et al: KDM4B-mediated epigenetic silencing of miRNA-615-5p augments RAB24 to facilitate malignancy of hepatoma cells. Oncotarget 8: 17712-17725, 2017.

37. Bai Y, Li J, Li J, Liu Y and Zhang B: MiR-615 inhibited cell proliferation and cell cycle of human breast cancer cells by suppressing of AKT2 expression. Int J Clin Exp Med 8: 3801-3808, 2015.

38. Gao W, Gu Y, Li Z, Cai H, Peng Q, Tu M, Kondo Y, Shinjo K, Zhu Y, Zhang J, et al: miR-615-5p is epigenetically inactivated and functions as a tumor suppressor in pancreatic ductal adenocarcinoma. Oncogene 34: 1629-1640, 2015.

39. Heidegger I, Pircher A, Klocker H and Massoner P: Targeting the insulin-like growth factor network in cancer therapy. Cancer Biol Ther 11: 701-707, 2011.

40. Rainier S, Johnson LA, Dobry CJ, Ping AJ, Grundy PE and Feinberg AP: Relaxation of imprinted genes in human cancer. Nature 362: 747-749, 1993.

41. Chen W, Feng Y, Zhao Q, Zhu Z and Dimitrov DS: Human monoclonal antibodies targeting nonoverlapping epitopes on insulin-like growth factor II as a novel type of candidate cancer therapeutics. Mol Cancer Ther 11: 1400-1410, 2012.

42. Ji Y, Wang Z, Li Z, Huang N, Chen H, Li B and Hui B: Silencing IGF-II impairs C-myc and N-ras expressions of SMMC-7721 cells via suppressing FAK/PI3K/Akt signaling pathway. Cytokine 90: $44-53,2017$ 